\title{
Claves para la participación de los adolescentes en agrupaciones
} musicales juveniles: el caso de los coros en Catalunya y las bandas en Valencia

Diego Calderón Garrido

https://orcid.org/0000-0002-2860-6747

Universitat de Barcelona, Dep. Didácticas Aplicadas

dcalderon@ub.edu

\author{
Salvador Oriola Requena \\ https://orcid.org/0000-0002-2205-0020 \\ Universitat de Barcelona, Dep. Didácticas Aplicadas \\ salvaoriola@ub.edu \\ Josep Gustems Carnicer \\ https://orcid.org/0000-0002-6442-9805 \\ Universitat de Barcelona, Dep. Didácticas Aplicadas \\ jgustems@ub.edu \\ SCIENTIFIC ARTICLE \\ Submitted date: 27 jan 2021 \\ Final approval date: 13 jun 2021
}

Resumen: La práctica musical colectiva durante la adolescencia aporta un sinfín de beneficios tanto académicomusicales como socioemocionales. En España, existen dos fenómenos musicales asociativos que destacan por su calidad musical y su amplia oferta en educación no formal: las bandas sinfónicas en Valencia y las corales en Cataluña. La presente investigación consiste en un estudio exploratorio no experimental cuyo principal objetivo fue conocer las razones por las que los adolescentes deciden participar en dichas agrupaciones y los beneficios socioemocionales que perciben. Para ello se aplicó un cuestionario ad hoc a una muestra de 660 adolescentes. Los resultados obtenidos relacionaron la práctica musical colectiva con factores como el placer estético o la relación entre amigos. Se observaron diferencias estadísticamente significativas dependiendo de la tipología de agrupación. Los participantes valoran muy positivamente el liderazgo ejercido por el director musical, así como los beneficios socioemocionales que obtienen.

Palabras clave: Adolescencia; Banda sinfónica; Coral; Beneficios socioemocionales; Participación.

\section{TITLE: KEYS FOR THE PARTICIPATION OF ADOLESCENTS IN YOUTH MUSICAL GROUPS: THE CASE OF CHOIRS IN CATALONIA AND BANDS IN VALENCIA}

Abstract: Group music practice during the adolescence brings many academic-music and socio-emotional benefits. In Spain, there are two associative musical phenomena that stand out for their musical quality and their wide offer of non-formal education: symphonic bands in Valencia and choirs in Catalonia. The present investigation consists of a non-experimental exploratory study whose main objective was to know the reasons why adolescents decide to participate in these groups and the socio-emotional benefits they perceive. To achieve this goal, an ad hoc questionnaire was applied to a sample of 660 adolescents. The results obtained related the group music practice with factors such as aesthetic pleasure or the relationship between friends. Statistically significant differences were observed depending on the type of grouping. Participants highly value the leadership exercised by the musical director, as well as the social-emotional benefits they obtain.

Keywords: Adolescence; Concert band; Choir; Socio-emotional benefits; Participation. 


\section{Claves para la participación de los adolescentes en agrupaciones musicales juveniles: el caso de los coros en Catalunya y las bandas en Valencia}

Salvador Oriola Requena, Universitat de Barcelona, salvaoriola@ub.edu Diego Calderón Garrido, Universitat de Barcelona, dcalderon@ub.edu Josep Gustems Carnicer, Universitat de Barcelona, jgustems@ub.edu

\section{Introducción}

La adolescencia es un periodo vital muy dinámico caracterizado por la sucesión de cambios personales significativos, tanto físicos como cognitivos. Esta idea ha sido desarrollada a lo largo del siglo XX y XXI, puesto que con anterioridad apenas se tenía en cuenta esta etapa preparatoria a la juventud. Así mismo, la vivencia de este periodo no es uniforme en todas las culturas y sociedades, por lo que muchos expertos prefieren destacar los cambios madurativos que van apareciendo para quedarse en la forma adulta de conducta, lo que ha venido a denominarse "madurez emergente" (Arnett 2008). En estos cambios influirán elementos tan diversos como las particularidades de cada persona, su formación, el grupo de iguales, la familia, el contexto más cercano, las nuevas tecnologías, los intereses personales, etc. Para hacer frente a todo ello de forma exitosa, las personas deberán adquirir y desarrollar un sinfín de estrategias, capacidades y competencias que les permitan realizar los ajustes y las adaptaciones necesarias (Berger 2007). Así pues, no hay una única adolescencia, sino adolescencias y adolescentes puesto que cada uno de estos establecerá un tipo de relaciones socioemocionales determinadas, vivirá un proceso de maduración único, adquirirá unos valores específicos, etc., los cuales serán diferentes en cada caso (Castillo, 1999).

Durante la adolescencia se consolida el pensamiento formal abstracto que permite a las personas empezar a ser conscientes y configurar su propia identidad tanto personal como social; sus gustos, intereses y valores; o su grupo de amigos y su sentido de pertenencia, entre otras cosas. El adolescente relega al grupo de amigos la función social que había desempeñado su familia hasta el momento (soporte, comprensión, pertenencia, etc.), se preocupa por su apariencia física y su identidad, su autoestima ante tantos cambios será fluctuante e irá estabilizándose con el paso del tiempo gracias a un mayor conocimiento y aceptación del propio cuerpo y del yo real. Para hacer frente a tanto dinamismo de forma eficiente será fundamental poseer y desarrollar competencias socioemocionales como conocer, expresar y regular sus emociones, automotivarse, relacionarse adecuadamente con los otros, etc. De ahí nuestro interés por conocer en la presente investigación aspectos relacionados con este tipo de competencias.

En las últimas décadas, el uso habitual de las nuevas tecnologías, la hiperconectividad o la sobreinformación son algunos de los fenómenos que han influido significativamente en todo el proceso descrito, 
contribuyendo de esta forma a cambiar la concepción tradicional que se tenía sobre la adolescencia (Feixa 2014). Los adolescentes del primer cuarto del siglo XXI, apodados con nombres dispares como Generación $Z$, \# Generación, postmilennials o centennials, son nativos digitales inherentes al aprendizaje virtual y a las comunicaciones instantáneas, de ahí que los dispositivos móviles (teléfonos, tabletas...) y los ordenadores sean elementos capitales en esta etapa vital. Dichas herramientas son el medio que les permite acceder a cualquier tipo de información siempre que lo deseen, estar conectados con sus amistades en todo momento y compartir sus inquietudes a través de las redes sociales (Vilanova y Ortega 2017).

En todo este contexto adolescente tan ambiguo y volátil, la funcionalidad de la música ocupará un lugar muy importante. Durante la adolescencia se descubre que el uso de la música en todas sus vertientes (percepción, práctica y creación) puede servir como estrategia para afrontar y adaptarse a los múltiples cambios citados. El adolescente puede utilizar la música como regulador emocional ante altibajos socioemocionales, muy comunes durante esta etapa vital; la música puede actuar como inhibidor de estados emocionales no deseados o despertar e intensificar emociones positivas (Oriola y Gustems 2016). También se utiliza habitualmente como recurso de ocio; los adolescentes a través de la música obtienen placer estético, entretenimiento y distracción, así pues no es de extrañar que el uso de nuevas tecnologías, la música y la práctica deportiva formen parte del ranking de actividades de ocio preferidas por los jóvenes en España (Ricoy y Fernández-Rodríguez 2016). Estrechamente relacionada con esta función de ocio también encontramos la función de acompañamiento de otras actividades; la generación Z se caracteriza por su faceta multitasking, por ello los jóvenes suelen acompañar con música tareas de todo tipo como por ejemplo estudiar, caminar o chatear (Miranda 2013). Otra función destacada que desempeña la música durante la adolescencia es su uso como recurso para la configuración y consolidación tanto de la propia identidad como de las relaciones interpersonales; las preferencias musicales se convierten en un aspecto personal muy destacado a través del cual, además de identificarse uno mismo y darse a conocer a los demás, también les sirve para conocer a gente nueva o relacionarse con iguales que compartan preferencias (Oriola y Gustems 2015).

Por la significatividad que ocupan todas estas funciones en la vida adolescente se puede afirmar que música y adolescencia forman un binomio inseparable que en la actualidad se ha acrecentado y fortalecido debido a la confluencia de diferentes razones. Por un lado, el desarrollo de las nuevas tecnologías ha permitido, tal y como se ha comentado, que cualquier adolescente a través de un dispositivo móvil pueda acceder a todo tipo de música desde cualquier lugar y siempre que lo desee. Por otro lado, la conjunción que se da entre dicho desarrollo tecnológico y la amplia oferta de educación no formal existente, también ha favorecido que el aprendizaje musical sea más accesible para todos. Durante las últimas décadas ha proliferado la educación musical no formal a través de escuelas de música, colonias musicales, clases particulares, etc. (Berbel Gómez y Díaz Gómez 2014), y también a través del uso de las nuevas tecnologías como cursos online, video tutoriales, etc. (Segura Jerez 2017). En este sentido, caben destacar los beneficios que aportan a los adolescentes los modelos de educación musical en contextos no formales, frente a la educación formal, de carácter más reglado y conservador. Mientras la primera se adapta a unas necesidades y desafíos sociales e individuales determinados, la otra queda muchas veces rezagada por su rigidez y generalización (Calderón, Gustems, y Calderón 2016).

En España destacan dos regiones con unos fenómenos asociativos modélicos por lo que a la oferta de educación musical no formal se refiere. Estos son las bandas y las corales en la Comunidad Valenciana y Catalunya, respectivamente. En ambas regiones existe una amplia tradición musical de más de un siglo de 
historia, fruto de ello son las más de mil agrupaciones musicales que acumulan entre ambos fenómenos (Federació de Societats Musicals de la Comunitat Valenciana 2020; Federació Catalana d'Entitats Corals 2020). En lo que se refiere a la formación de los adolescentes, dichas agrupaciones suelen tener secciones juveniles que, en muchos casos, además de proporcionar aprendizajes musicales sirven como preparación para acceder a agrupaciones de adultos con un nivel artístico superior.

Corales y bandas juveniles comparten algunos rasgos tanto a nivel idiosincrático como a nivel formativo. Ambos tipos de agrupaciones desempeñan una importante tarea socio-cultural en su contexto más próximo y por ello son consideradas, por parte de la sociedad donde están inmersas, como símbolos culturales e identitarios (Carbonell 2000; Leal 2014). En cuanto a la formación, corales y bandas, coinciden en ofrecer una educación musical de calidad, basada en la práctica musical colectiva. Por ello, los adolescentes integrantes de este tipo de agrupaciones, además de desarrollar competencias puramente musicales también adquieren otro tipo de aprendizajes estrechamente relacionados con la educación emocional.

Por lo que respecta a las competencias musicales, los componentes de estas agrupaciones aprenden a seguir las indicaciones del director musical, desarrollan su capacidad auditiva a través de la afinación con otros instrumentos o voces, toman conciencia sobre los diferentes planos sonoros, practican la sincronía rítmica colectiva, escuchan y desarrollan aspectos de carácter expresivo, etc. (Oriola, Gustems, y Filella 2019). Por otro lado, el aprendizaje emocional que se adquiere y desarrolla en el seno de dichas agrupaciones está vinculado con aspectos como la convivencia y comunicación entre todos los integrantes de la agrupación, el sentimiento de pertenencia, el aprendizaje entre iguales, el trabajo colectivo, la adquisición de valores, etc. (Calderón 2014; Ferrer, Puiggalí, y Tesouro 2018).

La adquisición y el trabajo de las citadas competencias puede extrapolarse a otros escenarios de la vida cotidiana de cada adolescente, y tal como demuestran diferentes investigaciones, puede repercutir positivamente en el desarrollo de otro tipo de aprendizajes y logros. Por un lado están los conocimientos de tipo más académico como las matemáticas (Schellenberg and Weiss 2013), la lectura (Southgate y Roscigno 2009), la motivación académica (Oriola-Requena, Gustems-Carnicer, y Filella-Guiu 2018) y, por otro, los aprendizajes intrapersonales como la conciencia y la regulación emocional o la empatía, entre otros aspectos (Ros-Morente et al. 2019).

En todo este proceso de aprendizaje colectivo, la figura del director musical ocupará un papel fundamental, independientemente de la tipología de agrupación. Este no solo será el encargado de transmitir conocimientos musicales sino también de establecer un buen clima de trabajo y unas buenas relaciones interpersonales, hacer partícipe a todos los integrantes de un objetivo común, ser empático y comprensible con las particularidades de cada músico, aprovechar las emociones que se experimentan tanto a nivel individual como colectivo, etc. (Oriola y Gustems 2019). Para ello deberá conocer, aplicar y combinar diferentes tipologías de liderazgo que se adapten a las características y necesidades de cada agrupación. Por ejemplo, si se quiere ser productivo y motivar a los integrantes de una agrupación reducida en la que el nivel musical sea básico, el director deberá aplicar un liderazgo organizativo que incluya, además de encabezar la práctica musical colectiva, la realización de arreglos musicales adaptados al número de participantes y a su nivel musical particular (Gustems, Calderón, y Oriola 2015).

Sin embargo, y a pesar de todas las semejanzas descritas y compartidas entre ambas tipologías de agrupaciones, también existen diferencias significativas como por ejemplo la formación musical mínima necesaria para acceder a cada una de ellas; en una banda juvenil se necesita de una formación básica (3-4 
años), en cambio esta formación no es un requisito sine qua non en las corales. Otro aspecto destacable son los recursos económicos y organizativos propios de cada tipología de agrupación; para crear y mantener una banda se necesita de unos instrumentos musicales y de unos profesores que enseñen la técnica de dichos instrumentos, lo cual implica una inversión económica y logística considerable, mientras que dicha inversión en las corales suele ser mucho más modesta.

Conocida la importancia que ocupa la música en la vida adolescente y partiendo de las semejanzas y diferencias descritas entre bandas y corales juveniles nos surgen las siguientes cuestiones ¿cuáles son las razones por las que los adolescentes deciden participar en una agrupación musical? ¿por qué en una banda y no en una coral, o viceversa? ¿qué es lo que más les gusta de la práctica musical colectiva? ¿perciben que mejora su perfil socioemocional? ¿qué opinión tienen sobre su director musical? Ante este tipo de cuestiones ¿existirán diferencias entre bandas y coros? En la presente investigación se intentará dar respuesta a todas estas cuestiones.

\section{Objetivos}

El objetivo principal de esta investigación es conocer las razones por las que los adolescentes deciden pertenecer a una agrupación musical juvenil (bandas y coros). Para alcanzar el objetivo citado se plantearon los siguientes objetivos específicos:

1. Conocer el motivo principal para formar parte de una agrupación musical.

2. Conocer qué es lo que más les gustaba a los participantes de su agrupación musical.

3. Conocer la valoración que hacen los participantes sobre la dirección musical: su relación interpersonal y su formación.

4. Conocer los beneficios socioemocionales percibidos por formar parte de una agrupación musical juvenil.

5. Conocer si se existen diferencias entre bandas y corales, entre hombres y mujeres, y detectar correlaciones entre los diferentes factores estudiados.

\section{Método}

Para dar respuesta a los objetivos planteados se diseñó un estudio transversal exploratorio no experimental, descriptivo ex post facto, consistente en la creación y aplicación de un cuestionario ad hoc en una muestra de adolescentes integrantes de una agrupación musical. Se optó por un estudio de corte cuantitativo debido al acceso obtenido a una amplia muestra de músicos adolescentes, por lo que se descartó recoger datos cualitativos para este estudio. Mediante el análisis de los datos cuantitativos obtenidos se pretende ofrecer una panorámica acerca de las razones por las que los adolescentes pertenecen a una agrupación musical y los beneficios emocionales que autoperciben por participar en ellas.

\subsection{Muestra}

La muestra del presente estudio está conformada por 660 músicos adolescentes (edad media 14.26 años, SD = 2.23), de los cuales 367 (55.6\%) pertenecían a una banda sinfónica juvenil de la Comunidad Valenciana (España), y los otros 293 (44.4\%) cantaban en una coral juvenil de Catalunya (España). De la muestra total, 
$237(35.9 \%)$ eran hombres, mientras que 423 (64.1\%) eran mujeres, distribuidos de la siguiente forma (ver figura 1):

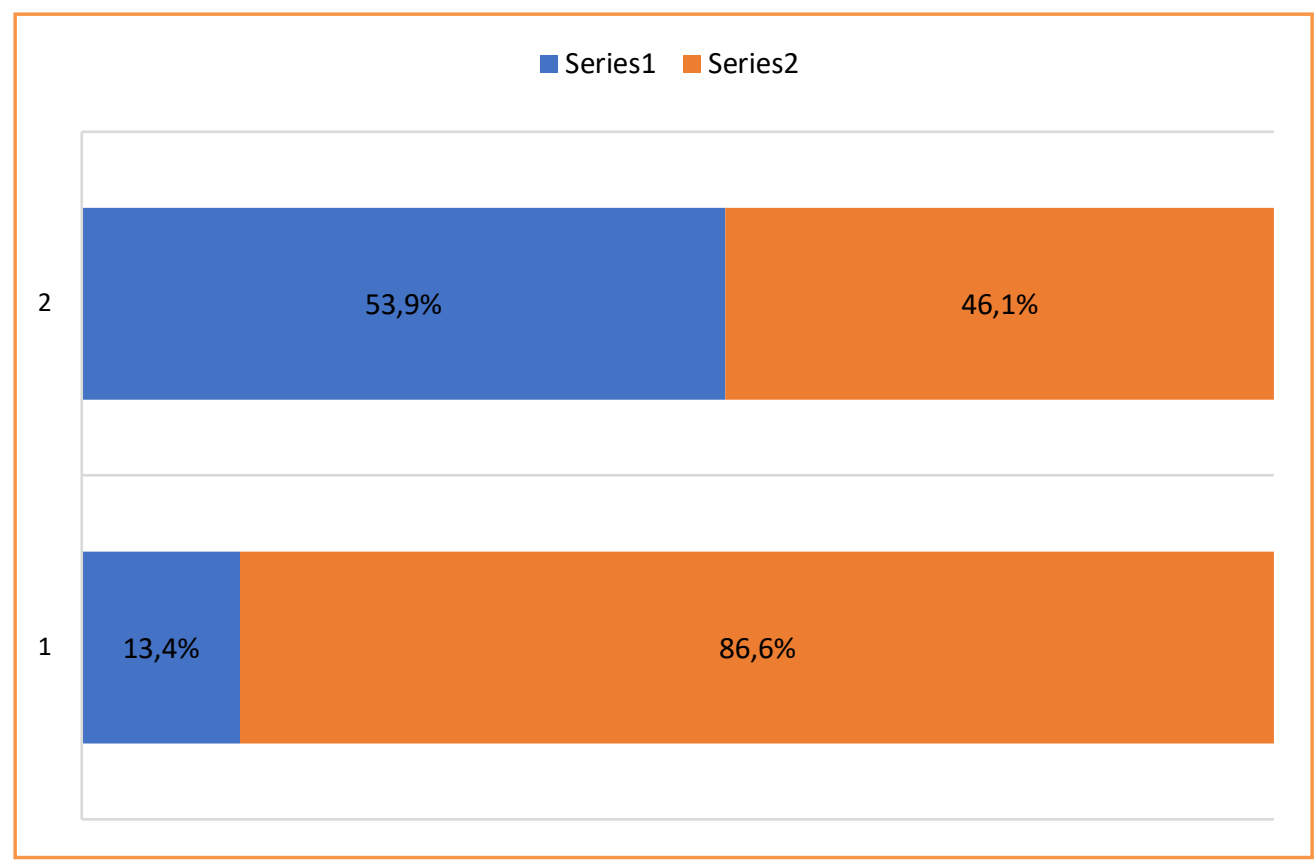

Figura 1 - Distribución del género según la tipología de agrupación

Pese a tratarse de una muestra no probabilística e intencional, la muestra empleada se puede considerar "representativa" de los fenómenos corales y bandísticos juveniles presentes en Catalunya y Valencia, respectivamente. En el presente estudio, los participantes procedían de 20 agrupaciones juveniles, 10 bandas y 10 corales, seleccionadas para resultar representativas de ambos territorios, para obtener así unos resultados que reflejen al máximo la realidad de ambos fenómenos asociativos.

\subsection{Instrumento}

El instrumento elegido para la recopilación de datos fue un cuestionario ad hoc que permitió abordar aspectos sociodemográficos así como auto informados relativos a las preferencias en la pertenencia a la agrupación musical y los beneficios socioemocionales percibidos.

La validación de dicho cuestionario se realizó a través de la técnica de juicio de expertos. Concretamente 10 expertos de diferentes disciplinas (profesores doctores de diferentes universidades españolas, directores de coros y bandas, profesores de educación secundaria y profesores de escuelas de música y conservatorios) se encargaron de realizar correcciones, objeciones y consejos sobre el cuestionario. Posteriormente se realizó una prueba piloto a 5 adolescentes instrumentistas y a 5 adolescentes cantores de agrupaciones juveniles para verificar su funcionalidad. Todo ello permitió eliminar y modificar algunas de las preguntas propuestas inicialmente e incorporar otras nuevas, por lo que la redacción del cuestionario definitivo ganó en especificidad, comprensibilidad, claridad y relevancia.

El cuestionario definitivo (ver Anexo) quedó configurado por un total de 13 ítems, en el que la mayoría de las respuestas eran cerradas o de elección múltiple, es decir, que para cada enunciado interrogativo se propuso una escala tipo Likert o una serie de respuestas entre las que el encuestado debía escoger aquélla o aquéllas que más se adaptaran a sus propias características. 


\subsection{Procedimiento}

Para la aplicación de los cuestionarios descritos, los investigadores establecieron un contacto inicial con 10 corales juveniles de Catalunya y 10 bandas de música juveniles de la Comunidad Valenciana a través de su director musical, al cual se informó de los detalles del estudio y se le encargó la tarea de transmitir la información a los componentes de su respectiva agrupación.

Aceptada la invitación por parte de la agrupación musical, al tratarse de menores de edad, se facilitó a los progenitores de los participantes un documento informativo acerca de la investigación y la autorización para aplicar a sus hijos/as los cuestionarios de forma voluntaria y anónima. Obtenido dicho consentimiento informado, un investigador se desplazó hasta el lugar de ensayo de cada agrupación y se encargó de aplicar el cuestionario en formato papel. Los datos obtenidos se trataron mediante códigos de identificación para preservar el anonimato y la confidencialidad de los resultados, siguiendo en todo momento los condicionantes del Informe Belmont (1978) y el Código de Buenas Prácticas en Investigación de la universidad a la que pertenecen los investigadores.

\subsection{Análisis}

Para el recuento y análisis estadístico de los resultados se utilizó el programa IBM Statistic Package for Social Science (SPSS), en su versión 21.0. En todos los casos se estableció un intervalo de confianza mínimo del 95\%. Además de los estadísticos descriptivos, se buscaron las diferencias estadísticas entre bandas y corales con los estadísticos de Mann-Whitney (en los casos con dos variables no paramétricas) y el de Kruskal-Wallis (en los casos con más de dos variables no paramétricas), realizando previamente las pruebas de normalidad de la muestra de Kolmogorov-Smirnov o de Shapiro-Wilk en función de las necesidades, así como el estadístico de Levene para comprobar la homogeneidad de varianzas, y el análisis de clúster de conglomerados con la prueba de esfericidad de Barlett.

\section{Resultados}

Respecto a la pregunta sobre los motivos para formar parte de una agrupación, la mayoría de los participantes consideraron que era porque les gustaba, aunque en sus respuestas se observaron diferencias estadísticas en función de la agrupación musical. Destaca que, en la banda, los participantes que están por su futuro profesional son un $12 \%$ más que en las corales. Todos los datos se pueden ver en la Tabla 1.

Tabla 1 - Razones para pertenecer a una agrupación musical y diferencias en función de la agrupación

\begin{tabular}{|c|c|c|c|c|c|c|}
\hline & Me gusta & $\begin{array}{c}\text { Futuro } \\
\text { profesional }\end{array}$ & Amistades & Obligación & Otras razones & $\begin{array}{l}\text { Diferencias } \\
\text { estadísticas }\end{array}$ \\
\hline $\begin{array}{l}\text { Bandas } \\
n=367\end{array}$ & $\begin{array}{c}285 \\
(77.7 \%) \\
\end{array}$ & $\begin{array}{c}71 \\
(19.3 \%)\end{array}$ & $\begin{array}{c}8 \\
(2.2 \%)\end{array}$ & $\begin{array}{c}1 \\
(.3 \%)\end{array}$ & $\begin{array}{c}2 \\
(.5 \%)\end{array}$ & \multirow{2}{*}{$\begin{array}{c}Z=-4.012 \\
p<.001\end{array}$} \\
\hline $\begin{array}{l}\text { Corales } \\
n=293\end{array}$ & $\begin{array}{c}254 \\
(86.7 \%)\end{array}$ & $\begin{array}{c}21 \\
(7.2 \%)\end{array}$ & $\begin{array}{c}11 \\
(3.8 \%)\end{array}$ & $\begin{array}{c}4 \\
(1.4 \%)\end{array}$ & $\begin{array}{c}3 \\
(1 \%)\end{array}$ & \\
\hline $\begin{array}{l}\text { Total } \\
n=660\end{array}$ & $\begin{array}{c}539 \\
(81.7 \%)\end{array}$ & $\begin{array}{c}92 \\
(13.9 \%)\end{array}$ & $\begin{array}{c}19 \\
(2.9 \%)\end{array}$ & $\begin{array}{c}5 \\
(.8 \%)\end{array}$ & $\begin{array}{c}5 \\
(.8 \%)\end{array}$ & \\
\hline
\end{tabular}

En la descrita motivación, no se detectaron diferencias estadísticas en función del género $(Z=1.945 ; p=$ .052). 
Por otro lado, respecto a la pregunta sobre qué es lo que más les gustaba a los participantes de su agrupación musical, la respuesta preferida fue la relación con los compañeros. Sin embargo, destacó la diferencia entre bandas y corales. Además, en lo relativo a la relación con los compañeros (más habitual en las corales), fue remarcable que los conciertos fue la respuesta preferida en $17 \%$ más de casos en bandas que en corales. Estas preferencias no estaban ligadas a la antigüedad en la formación $\left(X^{2}{ }_{3}=7.039 ; p=.071\right)$. Todos estos datos se pueden observar en la Tabla 2.

Tabla 2 - Qué se valora más en una agrupación musical y diferencias entre grupos

\begin{tabular}{|c|c|c|c|c|c|c|}
\hline & $\begin{array}{l}\text { Relación con } \\
\text { compañeros }\end{array}$ & Conciertos & $\begin{array}{l}\text { Conocer } \\
\text { repertorio }\end{array}$ & Ensayos & Otras razones & $\begin{array}{l}\text { Diferencias } \\
\text { estadísticas }\end{array}$ \\
\hline $\begin{array}{l}\text { Bandas } \\
n=367\end{array}$ & $\begin{array}{c}183 \\
(49.9 \%)\end{array}$ & $\begin{array}{c}137 \\
(37.3 \%)\end{array}$ & $\begin{array}{c}22 \\
(6 \%)\end{array}$ & $\begin{array}{c}25 \\
(6.8 \%)\end{array}$ & 0 & \multirow{2}{*}{$\begin{array}{c}Z=-3.534 \\
p<.001\end{array}$} \\
\hline $\begin{array}{l}\text { Corales } \\
n=293\end{array}$ & $\begin{array}{c}194 \\
(66.2 \%)\end{array}$ & $\begin{array}{c}59 \\
(20.1 \%)\end{array}$ & $\begin{array}{c}19 \\
(6.5 \%)\end{array}$ & $\begin{array}{c}14 \\
(4.8 \%)\end{array}$ & $\begin{array}{c}7 \\
(2.4 \%)\end{array}$ & \\
\hline $\begin{array}{l}\text { Total } \\
n=660\end{array}$ & $\begin{array}{c}377 \\
(57.1 \%)\end{array}$ & $\begin{array}{c}196 \\
(29.7 \%)\end{array}$ & $\begin{array}{c}41 \\
(6.2 \%)\end{array}$ & $\begin{array}{c}39 \\
(5.9 \%)\end{array}$ & $\begin{array}{c}7 \\
(1.1 \%)\end{array}$ & \\
\hline
\end{tabular}

No se reportaron diferencias en función del género a la hora de especificar que es lo que a los integrantes de las agrupaciones más les gustaba $(Z=.401 ; p=.689)$.

Respecto a la dirección musical, la formación musical de los directores estuvo muy bien valorada $(M=9.05$; $S D=1.01)$. En este sentido, no se observaron diferencias estadísticas en función del género $(Z=.432 ; p=$ .666; Hombres: $M=8.72 ; S D=1.12$; Mujeres: $M=8.98 ; S D=1.24)$. Sin embargo, sí se reportaron diferencias estadísticas significativas en función de la agrupación, siendo mucho más valorada en las corales que en las bandas ( $Z=-4.183 ; p<.001$; Bandas: $M=8.90 ; S D=1.07$; Corales: $M=9.24 ; S D=.88$ ).

Por lo que se refiere a la relación personal con su director, fue igualmente muy bien valorada $(M=8.30 ; S D$ = 1.32). Al igual que en el caso anterior, no se observaron diferencias estadísticamente significativas en función del género $(Z=1.366 ; p=.172$; Hombres: $M=8.20 ; S D=.95$; Mujeres: $M=8.36 ; S D=1.09)$ pero sí en función de la agrupación. Dicha relación fue mejor valorada en las corales que en las bandas, observándose diferencias estadísticas significativas $(Z=-2.976 ; p=.003$; Bandas: $M=8.19 ; S D=1.32$; Corales: $M=8.45 ; S D$ $=1.38$ ). Se observó una correlación entre la formación musical del director y la relación con este ( $r=.442 ; p$ $<$.001). Así pues, esta buena relación estaba relacionada con lo que los músicos opinaban sobre su formación.

Respecto a los beneficios socioemocionales que los participantes autopercibían por pertenecer a una agrupación musical, los resultados mostraron como, tanto conocer las emociones propias como las de los de los compañeros, aprender a expresar y regular las emociones, la mejora de la autoestima, la mejora de las relaciones con los otros, y, en especial, la mejora del bienestar, fueron aspectos valorados muy positivamente, mejor en el caso de las bandas que en las corales. No se detectaron diferencias estadísticas significativas en función del género en ninguno de los casos (en el caso de conocer las emociones $Z=.288 ; p$ $=.773$, respecto a expresar y regular las emociones $Z=.164 ; p=.869$, por lo que se refiere a mejorar la autoestima $Z=.081 ; p=.935$, en el caso de mejorar el bienestar $Z=1.45 ; p=.072$, respecto a la ayuda en la relación con otros $Z=1.935 ; p=.053$ ). 
Todos estos ítems mostraron una alta correlación entre sí. Así mismo, el análisis de conglomerados (prueba de esfericidad de Barlett $p<.001$ ) de las respuestas obtenidas mostró que los participantes que hacían alusión al conocimiento de las emociones también lo hacían a la expresión y regulación de estas. Todos los datos se pueden observar en la Tabla 3.

Tabla 3 - Beneficios socioemocionales autopercibidos en función de la agrupación musical y correlación entre los resultados

\begin{tabular}{|c|c|c|c|c|c|c|c|c|c|}
\hline & \multirow{2}{*}{$\begin{array}{c}M \\
(S D)\end{array}$} & \multirow{2}{*}{ Bandas } & \multirow{2}{*}{ Corales } & \multirow{2}{*}{$\begin{array}{l}\text { Diferencias } \\
\text { estadísticas }\end{array}$} & \multicolumn{5}{|c|}{ Correlaciones } \\
\hline & & & & & 1. & 2. & 3. & 4. & 5. \\
\hline $\begin{array}{l}\text { 1. Conocer las } \\
\text { emociones }\end{array}$ & $\begin{array}{c}7.57 \\
(1.65)\end{array}$ & $\begin{array}{c}7.65 \\
(1.60)\end{array}$ & $\begin{array}{c}7.47 \\
(1.69)\end{array}$ & $\begin{array}{c}Z=-1.30 \\
p=.194\end{array}$ & 1 & & & & \\
\hline $\begin{array}{l}\text { 2. Expresar y } \\
\text { regular las } \\
\text { emociones }\end{array}$ & $\begin{array}{c}7.95 \\
(1.62)\end{array}$ & $\begin{array}{c}8.14 \\
(1.47)\end{array}$ & $\begin{array}{c}7.71 \\
(1.76)\end{array}$ & $\begin{array}{c}Z=-2.871 \\
p=.004\end{array}$ & $.614^{* *}$ & 1 & & & \\
\hline $\begin{array}{l}\text { 3. Mejora mi } \\
\text { autoestima }\end{array}$ & $\begin{array}{c}7.77 \\
(1.39)\end{array}$ & $\begin{array}{c}7.99 \\
(1.30)\end{array}$ & $\begin{array}{c}7.49 \\
(1.46)\end{array}$ & $\begin{array}{c}Z=-4.383 \\
p<.000\end{array}$ & $.348^{* *}$ & $.429^{* *}$ & 1 & & \\
\hline $\begin{array}{l}\text { 4. Mejora mi } \\
\text { bienestar }\end{array}$ & $\begin{array}{c}8.66 \\
(1.42)\end{array}$ & $\begin{array}{c}8.82 \\
(1.29)\end{array}$ & $\begin{array}{c}8.46 \\
(1.55)\end{array}$ & $\begin{array}{c}Z=-2.881 \\
p=.004\end{array}$ & $.413^{*}$ & $.439^{* *}$ & $.495^{* *}$ & 1 & \\
\hline $\begin{array}{l}\text { 5. Ayuda en la } \\
\text { relación con } \\
\text { otros }\end{array}$ & $\begin{array}{c}7.93 \\
(1.37)\end{array}$ & $\begin{array}{c}8.07 \\
(1.65)\end{array}$ & $\begin{array}{c}7.75 \\
(1.38)\end{array}$ & $\begin{array}{c}Z=-3.024 \\
p=.002\end{array}$ & $.400^{* *}$ & $.337^{* *}$ & $.378^{* *}$ & $.362^{* *}$ & 1 \\
\hline
\end{tabular}

$* * \mathrm{p}<.001$

\section{Discusión}

Los resultados obtenidos confirman que los adolescentes que participan en una agrupación musical juvenil, independientemente de si es un coro o una banda sinfónica, lo hacen principalmente porque les gusta. Y ¿por qué les gusta? Porque tal y como afirman diferentes estudios (Rawlings y Stoddard 2017; Parker 2020), la práctica musical colectiva, además de placer estético, también aporta una experiencia compartida entre iguales y amigos, desarrolla el sentido de pertenencia y aceptación en un grupo, la sensación de sentirse competente, o la configuración de la propia identidad tanto individual como social. Todos estos elementos ocupan un lugar muy relevante en la vida adolescente puesto que, en una etapa de metamorfosis y sus correspondientes adaptaciones vitales, contribuyen al aumento de satisfacción y bienestar personal.

Sin embargo, existe una diferencia significativa entre grupos en cuanto a las razones por pertenecer a este tipo de agrupaciones. Mientras que en las corales solo un $7.2 \%$ de los participantes indican pertenecer a ellas en vista a una posible salida laboral, en las bandas este porcentaje se eleva hasta el $19.3 \%$. Por ello se puede afirmar que un grupo destacado de integrantes de las bandas indica participar en ellas no solo por entretenimiento y placer sino con el objetivo de obtener una formación profesional que les ayude a poder dedicarse profesionalmente a la música en un futuro. Esta opción puede que se deba a las diferencias logísticas y de formación existentes entre ambos tipos de agrupaciones, especialmente las relacionadas con la formación musical requerida que incluye unos conocimientos teórico-musicales e instrumentales mínimos para poder interpretar eficazmente las partituras exigidas por su agrupación. Los alumnos instrumentistas, después de unos años de aprendizaje (2-4 años) se incorporan a sus respectivas bandas juveniles, a una edad que suele oscilar entre los 10 y 12 años (Oriola, Gustems, y Filella 2019).

En la misma línea siguen los resultados sobre los factores mejor valorados en ambos tipos de agrupaciones. Según los resultados obtenidos, la relación con los compañeros es aquello que más les gusta de la práctica musical colectiva, todo ello coindice con otros estudios según los cuales en este tipo agrupaciones existe una notable cohesión social fruto de la confluencia de elementos diversos como el trabajo en equipo, las 
relaciones interpersonales con personas de "ideas y gustos afines" o el sentimiento de pertenencia (North, Hargreaves, y O'Neill 2000; Kokotsaki, y Hallam 2007). Esto se da sobre todo en las corales, ya que según su idiosincrasia, en muchas de estas agrupaciones la vertiente musical y la vertiente social tienen un peso similar, mientras que en las bandas suele predominar la primera (Oriola, Gustems, y Filella 2018), tal y como reflejan el 37.3\% de los integrantes de banda, que indican los conciertos como opción preferida frente al $20.1 \%$ de las corales.

En cuanto a la figura del director, en ambos tipos de agrupaciones se valoró muy positivamente tanto la formación como el trato personal con los integrantes. No obstante, se encontraron diferencias entre grupos, siendo los integrantes de las corales quienes mejor valoran el trabajo llevado a cabo por sus respectivos directores. De acuerdo con diferentes estudios (Fernández, Corraliza, y Ferreras 2017; Silvey y Fisher 2015), los perfiles de los directores de agrupaciones musicales varían según si son coros o bandas. En las bandas predominan varones cuya edad no supera los 40 años, están en posesión de un título superior de música, y dirigen una agrupación juvenil con el objetivo de adquirir experiencia que les permita acceder en un futuro a dirigir una banda de adultos. En cambio, el perfil de director de coral es mucho más heterogéneo, existe paridad de género entre directores y directoras, pero sus edades y su formación musical son dispares. De acuerdo con lo expuesto, el director de banda juvenil, pese a tener una mayor formación académico-musical, desempeña una tarea en muchos casos de carácter transitorio mientras que los directores de coral suelen permanecer más tiempo al frente de una agrupación cosa que les permite conocer con más detalles el funcionamiento de este tipo de agrupaciones y el perfil de sus integrantes (Oriola, Gustems, y Elgström 2018), tal y como demuestra la valoración de la muestra.

Por otro lado, encontramos que los adolescentes participantes autoperciben recibir unos beneficios socioemocionales por el hecho de pertenecer a una agrupación musical. Esta autopercepción va en la línea de otros estudios según los cuales la participación de los jóvenes en una agrupación musical contribuye a la mejora de su alfabetización emocional y por consiguiente al aumento de su bienestar (Miranda 2013; Calderón 2014). Las bandas obtienen mejores puntuaciones que las corales en todos los factores socioemocionales investigados, aunque se observa una diferencia más notable en la autoestima y bienestar. Todo ello puede que tenga alguna relación con la disparidad de género según el tipo de agrupación, tal y como indican algunos estudios llevados a cabo con muestras adultas (Sánchez et. al. 2008).

\section{Conclusión}

En conclusión, la adolescencia es una etapa llena de cambios y reafirmación personales en la que se transita por la etapa infantil y se abre una puerta hacia la madurez. Esos años están llenos de inseguridades y miedos a ser diferente, a mostrase tal cual es cada uno. Se trata de un periodo en el que los modelos a seguir cambian, perdiendo protagonismo las familias en pro de los iguales, creándose así tensiones entre quién se es y quien se quiere ser. En este tránsito, tal como se ha observado en este estudio, los adolescentes encuentran en la práctica musical un espacio que convierten en su zona de confort. Una zona compartida que refuerza la pertinencia a un grupo de iguales, dando, si cabe más sentido a ese "iguales" en un clima de bienestar y entretenimiento que refuerza las competencias interpersonales de sus integrantes.

Si bien esa práctica musical puede suponer una salida laboral, tal pretensión de futuro es minoritaria, especialmente en lo referido a los músicos pertenecientes a corales juveniles. Sin embargo, esto no ha sido en detrimento de la motivación e interés por el placer y el resultado estético, ya que dicho interés también ha sido observado en ambas agrupaciones, sobre todo en el caso de las bandas. 
Por otro lado, se ha observado como la figura del director resulta crucial. En el caso de las agrupaciones juveniles, más allá del resultado musical, el liderazgo que ejerza ha de buscar una relación personal con cada uno de sus integrantes para que de esta forma encuentren en dicho director una motivación más a la hora de acudir a los ensayos. En el caso de la muestra estudiada, esto ha sido así.

Este estudio presenta una serie de limitaciones que, a su vez, se formulan como futuras líneas de investigación. Por un lado, si bien la muestra elegida es representativa, no deja de pertenecer únicamente a dos Comunidades Autónomas españolas, por lo que ampliar la muestra y los territorios se presenta como una oportunidad de conocer más a fondo los beneficios de pertenecer a una banda o coral juvenil. Por otro lado, otra línea de investigación que se abre es la de aumentar el tipo de agrupaciones, incluyendo a orquestas e incluso a grupos de música moderna, trabajen o no con un director musical. De esta forma se obtendría una visión global de lo que significa hacer música en grupo en la etapa adolescente y de las motivaciones que sus participantes encuentran en la pertenencia a cada uno de esos colectivos. También somo conscientes que pese a presentar una foto panorámica, los fenómenos sociales descritos son muy heterogéneos y por ello al trabajar con datos cuantitativos posiblemente dejemos al margen matices y detalles importantes que, con la incorporación de otro tipo de instrumentos como las entrevistas, podrían contribuir a una mejor y más detallada comprensión de la importancia social de las bandas y coros, permitiendo así trazar nuevas vías de pensamiento acerca del tema de estudio. Sin duda, una combinación de métodos y datos, lo que ha venido a llamarse mixed methods sería una buena manera de superar tales limitaciones.

En definitiva, en este estudio se han mostrado las razones que llevan a los adolescentes a hacer música en grupo y, lo que puede ser más significativo, todos los beneficios que ello comporta. Vistos estos resultados tan positivos, solo nos queda preguntarnos cuál es la razón por la que no todos los adolescentes participan aún en estas actividades. Esa ha de ser la misión de docentes, progenitores, investigadores, administraciones educativas, etc.: llevar la práctica musical a cada uno de nuestros menores con el fin de contribuir a su desarrollo integral.

\section{Referencias}

Arnett, Jeffrey Jensen. 2008. Adolescencia y Adultez Emergente. México: Pearson.

Berbel Gómez, Noemy, y Maravillas Díaz Gómez. 2014. “Educación Formal y No Formal. Un Punto de Encuentro En Educación Musical." Aula Abierta 42 (1): 47-52. doi:10.1016/S0210-2773(14)70008-3.

Berger, Kathleen Stassen. 2007. Psicología Del Desarrollo. Infancia y Adolescencia. Madrid: Médica Pan.

Calderón, Diego. 2014. “La Práctica En Grupo Como Camino Hacia El Bienestar de Los Adolescentes." En Arte y Bienestar, editado por Josep Gustems, 75-83. Barcelona: Universitat de Barcelona.

Calderón, Diego, Josep Gustems, y Caterina Calderón. 2016. "Projects in Music Education: A Bridge to WellBeing." Ponte. International Scientific Researches Journal 72 (4): 68-74. doi:10.21506/j.ponte.2016.4.9.

Carbonell, Jaume. 2000. "El Cant Coral." En Història de La Música Catalana, Valenciana i Balear. Del Romanticisme Al Nacionalisme, Vol. 3, editado por Xosé Aviñoa, 147-186. Barcelona: Edicions 62.

Castillo, Gerardo. 1999. El adolescente y sus retos. La aventura de hacerse mayor. Madrid: Pirámide.

Federació Catalana d'Entitats Corals. 2020. "Portal de La Federació Catalana d'Entitats Corals." https://www.fcec.cat/. 
Federació Societats Musicals de la Comunitat Valenciana. 2020. "Portal de La Federació Societats Musicals de La Comunitat Valenciana." https://fsmcv.org/.

Feixa, Carles. 2014. De La Generación@ a La \#Generación. Barcelona: NED Edició.

Fernández, Nuria, José Antonio Corraliza, y Soledad Ferreras. 2017. "Las Agrupaciones Corales En España: Espacios Para La Convivencia y La Educación Musical." Revista Internacional de Educación Musical 5: 17-20. doi:10.12967/RIEM-2017-5-p017-029.

Ferrer, Rita, Joan Puiggalí, y Montse Tesouro. 2018. "Choral Singing and the Acquisition of Educational Values." International Journal of Music Education 36 (3): 334-346. doi:10.1177/0255761417741521.

Gustems, Josep, Diego Calderón, y Salvador Oriola. 2015. "Music and Leadership: The Role of the Conductor." International Journal of Music and Performing Arts 3 (1): 84-88. doi:10.15640/ijmpa.v3n1a8.

Kokotsaki, Dimitra, y Susan Hallam. 2007. "Higher Education Music Students' Perceptions of the Benefits of Participative Music Making." Music Education Research 9 (1): 93-109. doi:10.1080/14613800601127577.

Miranda, Dave. 2013. "The Role of Music in Adolescent Development: Much More than the Same Old Song." International Journal of Adolescence and Youth 18 (1): 5-22. doi:10.1080/02673843.2011.650182.

North, Adrian C., David J. Hargreaves, y Susan A. O'Neill. 2000. "The Importance of Music to Adolescents." British Journal of Educational Psychology 70: 255-272. doi:10.1348/000709900158083.

Oriola-Requena, Salvador, Josep Gustems-Carnicer, y Gemma Filella-Guiu. 2018. "Agrupaciones Musicales Juveniles: Modelos Funcionales Para La Motivación Académica de Los Adolescentes." REIRE. Revista d'Innovació i Recerca En Educació 11 (2): 18-30. doi:10.1344/reire2018.11.220577.

Oriola, Salvador, y Josep Gustems. 2015. "Música Y Adolescencia: Usos, Funciones Y Consideraciones Educativas." Universitas Tarraconensis. Revista de Ciències de l'Educació 1 (2): 27-44. doi:10.17345/ute.2015.2.660.

Oriola, Salvador, y Josep Gustems. 2016. “El Procés Emocional d'escoltar i Produir Música." Temps d’Educació 50: 69-85.

Oriola, Salvador, y Josep Gustems. 2019. “La Dirección Musical de Agrupaciones Juveniles : Un Referente de Liderazgo y Competencias Musical Conducting of Youth Ensembles : A Benchmark for Leadership and Social-Emotional La Dirección Musical de Agrupaciones Juveniles : Un Referente de Liderazgo Y." Revista Internacional de Educación Musical 7: 69-79. doi:10.1177/2307484119878639.

Oriola, Salvador, Josep Gustems, y Edmon Elgström. 2018. "Perfil Profesional de Los Directores de Bandas y Corales Juveniles." Sonograma, no. 40.

Oriola, Salvador, Josep Gustems, y Gemma Filella. 2018. "Las Bandas y Corales Juveniles Como Recurso Para El Desarrollo Integral de Los Adolescentes." Revista Electrónica Complutense de Investigación En Educación Musical - RECIEM 15: 153-173. doi:10.5209/reciem.58813.

Oriola, Salvador, Josep Gustems, y Gemma Filella. 2019. “Las Bandas Juveniles de La Comunidad Valenciana: Medio Siglo Promoviendo La Educación Musical No Formal y Desarrollando Competencias Socioemocionales." ARTSEDUCA. Revista Electrónica de Educación En Las Artes 23: 36-51. doi:10.6035/artseduca.2019.23.2.

Parker, Elizabeth Cassidy. 2020. Adolescents on Music. Oxford: Oxford University Press. 
Rawlings, Jared R, y Sarah A Stoddard. 2017. "Peer Connectedness in the Middle School Band Program." Research Studies in Music Education 39 (1): 121-135. doi:10.1177/1321103X17703575.

Ricoy, María-Carmen, y Jennifer Fernández-Rodríguez. 2016. "Prácticas y Recursos de Ocio En La Adolescencia." Educatio Siglo XXI 34 (2): 103-124. doi:10.6018/j/263831.

Ros-Morente, Agnès, Salvador Oriola-Requena, Josep Gustems-Carnicer, y Gemma Filella Guiu. 2019. "Beyond Music: Emotional Skills and Its Development in Young Adults in Choirs and Bands." International Journal of Music Education 37 (4): 536-546. doi:10.1177/0255761419853634.

Sánchez, $M^{a}$ Trinidad, Pablo Fernández-Berrocal, Juan Montañés, y Jose Miguel Latorre. 2008. “¿Es La Inteligencia Emocional Una Cuestión de Género? Socialización de Las Competencias Emocionales En Hombres y Mujeres y Sus Implicaciones." Electronic Journal of Research in Educational Psychology 6 (15): 455-474.

Schellenberg, E. Glenn, y Michael W. Weiss. 2013. "Music and Cognitive Abilities." En The Psychology of Music, editado por Diana Deutsch, 499-550. Amsterdam: Elsevier.

Segura Jerez, Sonia. 2017. "Tecnologías de La Información y La Comunicación En El Aprendizaje Musical." AV Notas: Revista de Investigación Musical, no. 2: 133-156.

Silvey, Brian A, y Ryan A Fisher. 2015. "Effects of Conducting Plane on Band and Choral Musicians' Perceptions of Conductor and Ensemble Expressivity." Journal of Research in Music Education 63 (3): 369-383. doi:10.1177/0022429415597888.

Southgate, Darby E., y Vincent J. Roscigno. 2009. "The Impact of Music on Childhood and Adolescent Achievementn." Social Science Quarterly 90 (1): 4-21.

Vilanova, Núria, y Iñaki Ortega. 2017. Generación Z: Todo Lo Que Necesitas Saber Sobre Los Jóvenes Que Han Dejado Viejos a Los Millennials. Barcelona: Plataforma. 


\section{Anexo}

1. Edad:

2. Sexo:
$\square$ Hombre
Mujer

3. Estudios generales que estás cursando en la actualidad:
E.S.O.
$\square$ Bachillerato
Ciclo formativo
Universidad
Ed. Primaria

4. Estudios musicales realizados en:

Escuela de música

Conservatorio

Autodidacta

Otros:

5. ¿Cuántos años llevas estudiando música?

6. ¿Qué instrumento o instrumentos sabes tocar?

Principal:

Secundario:

7. ¿Por qué formas parte de una coral/banda?
$\square$ Por obligación
$\square$ Porque te gustaría dedicarte profesionalmente a la música
Porque te gusta
Porque están tus amigos
$\square$ Otros:

8. ¿Cuántos años hace, en total, que eres miembro de una coral/banda?

9. ¿Qué es lo que más te gusta de tu coral/banda?
La relación con los compañeros
Los ensayos
Los conciertos y actos oficiales
Conocer nuevo repertorio
Otros:

10. ¿Cómo te motiva el repertorio que se interpreta en tu coral/banda? Valora marcando con una cruz la casilla del 0 (nada) al 10 (mucho).

\begin{tabular}{|l|l|l|l|l|l|l|l|l|l|l|}
\hline 0 & 1 & 2 & 3 & 4 & 5 & 6 & 7 & 8 & 9 & 10 \\
\hline
\end{tabular}

11. ¿Cómo es la relación personal que tienes con los compañeros/as de tu coral/banda? Valora marcando con una cruz la casilla del 0 (nada) al 10 (mucho).

\begin{tabular}{|l|l|l|l|l|l|l|l|l|l|l|}
\hline 0 & 1 & 2 & 3 & 4 & 5 & 6 & 7 & 8 & 9 & 10 \\
\hline
\end{tabular}

12. ¿Cómo es la relación personal que tienes con el director/a de tu coral/banda? Valora marcando con una cruz la casilla del 0 (nada) al 10 (mucho).

\begin{tabular}{|l|l|l|l|l|l|l|l|l|l|l|}
\hline 0 & 1 & 2 & 3 & 4 & 5 & 6 & 7 & 8 & 9 & 10 \\
\hline
\end{tabular}


13. Valora del $\mathbf{0}$ (nada) al $\mathbf{1 0}$ (mucho) los beneficios socioemocionales que consideras que te aporta el hecho de pertenecer a una coral/banda:

\begin{tabular}{|c|l|c|c|c|c|c|c|c|c|c|c|c|}
\hline $\mathbf{1}$ & $\begin{array}{l}\text { Me ayuda a conocer mejor mis } \\
\text { emociones y la de mis compañeros }\end{array}$ & 0 & 1 & 2 & 3 & 4 & 5 & 6 & 7 & 8 & 9 & 10 \\
\hline $\mathbf{2}$ & $\begin{array}{l}\text { Me ayuda a expresar y regular las } \\
\text { emociones }\end{array}$ & 0 & 1 & 2 & 3 & 4 & 5 & 6 & 7 & 8 & 9 & 10 \\
\hline $\mathbf{3}$ & $\begin{array}{l}\text { Me motiva y me ayuda a mejorar mi } \\
\text { autoestima }\end{array}$ & 0 & 1 & 2 & 3 & 4 & 5 & 6 & 7 & 8 & 9 & 10 \\
\hline $\mathbf{4}$ & $\begin{array}{l}\text { Me ayuda a relacionarme con los } \\
\text { otros }\end{array}$ & 0 & 1 & 2 & 3 & 4 & 5 & 6 & 7 & 8 & 9 & 10 \\
\hline $\mathbf{5}$ & Me ayuda a mejorar mi bienestar & 0 & 1 & 2 & 3 & 4 & 5 & 6 & 7 & 8 & 9 & 10 \\
\hline
\end{tabular}

